\title{
SOME HESITANT FUZZY GEOMETRIC OPERATORS AND THEIR APPLICATION TO MULTIPLE ATTRIBUTE GROUP DECISION MAKING
}

\author{
Weize WANG ${ }^{\mathrm{a}}$, Xinwang $\mathrm{LIU}^{\mathrm{b}}$ \\ aSchool of Economics and Management, Guangxi Normal University, \\ Guilin, 541004 Guangxi, China \\ ${ }^{\mathrm{b} S}$ chool of Economics and Management, Southeast University, \\ Nanjing, 210096 Jiangsu, China
}

Received 10 March 2012; accepted 24 June 2012

\begin{abstract}
Hesitant fuzzy set (HFS), a generalization of fuzzy set (FS), permits the membership degree of an element of a set to be represented as several possible values between 0 and 1 . In this paper, motivated by the extension principle of HFs, we export Einstein operations on FSs to HFs, and develop some new aggregation operators, such as the hesitant fuzzy Einstein weighted geometric operator, hesitant fuzzy Einstein ordered weighted geometric operator, and hesitant fuzzy Einstein hybrid weighted geometric operator, for aggregating hesitant fuzzy elements. In addition, we discuss the correlations between the proposed aggregation operators and the existing ones respectively. Finally, we apply the hesitant fuzzy Einstein weighted geometric operator to multiple attribute group decision making with hesitant fuzzy information. Some numerical examples are given to illustrate the proposed aggregation operators.
\end{abstract}

Keywords: hesitant fuzzy set (HFS), Einstein t-norm, hesitant fuzzy Einstein weighted geometric $\left(\mathrm{HFWG}^{\varepsilon}\right)$ operator, hesitant fuzzy Einstein ordered weighted geometric $\left(\mathrm{HFOWG}^{\varepsilon}\right)$ operator, hesitant fuzzy Einstein hybrid weighted geometric $\left(\mathrm{HFHWG}^{\varepsilon}\right)$ operator, multiple attribute group decision making (MAGDM).

JEL Classification: C63, D81, L60, O33.

Corresponding author Weize Wang

E-mail:weizew@gmail.com 


\section{Introduction}

Aggregation operators, usually taking the forms of mathematical functions, are common techniques to fuse all the input individual data into a single one. Due to their great importance in the information processing of extensive areas, such as decision making, pattern recognition, information retrieval, medical diagnosis, data mining, machine learning, etc., the investigation on aggregation operators has been receiving much attention from both researchers and practitioners over the last decades (Yager, Kacprzyk 1997; Calvo et al. 2002; Xu, Da 2003; Torra, Narukawa 2007; Beliakov et al. 2007; Grabisch et al. 2009). Three of the most common geometric operators for aggregating arguments are the weighted geometric (WG) operator (Saaty 1980; Aczl, Saaty 1983; Willett, Sharda 1991; Benjamin et al. 1992; Xu 2000; Xu, Da 2003), the ordered weighted geometric (OWG) operator (Herrera et al. 2001; Chiclana et al. 2001; Xu, Da 2003), which based on the ordered weighted geometric (OWG) operator (Yager 1988) and the geometric mean, and the hybrid weighted geometric (HWG) operator (Xu, Da 2003). The WG operator first weights all the given arguments and then aggregates all these weighted arguments into a collective one. The fundamental aspect of the OWG operator is the reordering step before aggregating all the ordered weighted arguments into a collective one. The HWG operator generalizes both the OWG and WG operators and reflects the importance degrees of both the given argument and the ordered position of the argument.

In the real-life world, due to the increasing complexity of the socioeconomic environment and the lack of knowledge or data about the problem domain, crisp data are sometimes unavailable. Thus, the input arguments may be vague or fuzzy in nature. Besides fuzzy sets (FSs) by Zadeh (1965), several extensions of this concept have been introduced in the literature, for example, intuitionistic fuzzy sets (Atanassov 1986), interval-valued fuzzy sets (Zadeh 1973), type 2 fuzzy sets (Mizumoto, Tanaka 1976; Dubois, Prade 1980), fuzzy multisets (Yager 1986; Chakrabarty, Despi 2007) and hesitant fuzzy sets (HFSs) (Torra, Narukawa 2009; Torra 2010). IFSs are equivalent to interval-valued fuzzy sets (Atanassov, Gargov 1989; Cornelis et al. 2004). The membership of an element to a type 2 fuzzy set is defined in terms of a FS on the domain of memberships. IFSs can be seen, from a mathematical point of view, as a particular case of type 2 fuzzy sets (Dubois et al. 2005). Fuzzy multisets, or fuzzy bags, permit us to have multiple occurrences of the elements. The basic elements of a HFS are hesitant fuzzy elements (HFEs) (Xia, Xu 2011), each of which is characterized by a membership degree consisting of a set of possible values. Although all HFSs can be represented as fuzzy multisets, the operations on fuzzy multisets do not apply properly on HFSs. Torra and Narukawa (2009) and Torra (2010) showed that the envelope of HFS is an IFS, and proved that the operations applied to the envelope of HFS are consistent with the ones of IFS. It can be proved that HFSs can also be represented as type 2 fuzzy sets and IFS is a particular case of HFS.

In many practical situations, particularly in the process of group decision making under uncertainty and anonymity, the experts may come from different research areas and thus have different backgrounds and levels of knowledge, skills, experience, and personality, the experts may not have enough expertise or possess a sufficient level of knowledge to precisely express their preferences over the objects, and then, they usually have some uncertainty in providing 
their preferences. Moreover, the experts have only assigned a small and finite set in providing their preferences, where the difficulty may be caused by a doubt between a few different values. In such cases, the data or preferences given by the experts may be appropriately expressed in HFEs. For example, in multiple attribute group decision-making (MAGDM) problems, anonymity is required in order to protect the decision makers' privacy or avoid influencing each other, such as presidential election, blind peer review of thesis, etc., in which we do not know which attributes that the decision makers are respectively familiar with, and thus, leading us to consider all the situations in order to get more reasonable decision results. But the existing methods only consider the minor situations that each decision maker is good at evaluating all the attributes, which hardly happen. HFS is very useful in avoiding such issues in which each attribute can be described as a HFE defined in terms of the opinions of decision makers (Torra, Narukawa 2009). Up to now, some authors (Torra, Narukawa 2009; Torra 2010; Xu, Xia 2011a, b; Xia, Xu 2011; Xia et al. 2011; Rodriguez et al. 2012; Zhu et al. 2012a, b; Wei 2012; Yu et al. 2012) have paid attention to the HFS theory. Torra and Narukawa (2009) and Torra (2010) proposed the concept of HFS, which is deferent from other extensions exist for FSs, and also introduced some basic operations on HFSs. Torra and Narukawa (2009) presented an extension principle of HFSs, which permits to generalize existing operations on FSs to HFSs, and also discussed their use in decision making. Xu and Xia (2011a, b) proposed a variety of distance measures for HFSs, and particularly developed a number of hesitant ordered weighted distance measures and hesitant ordered weighted similarity measures, which can alleviate the influence of unduly large (or small) deviations on the aggregation results by assigning them low (or high) weights. Xia and Xu (2011) developed a series of aggregation operators for hesitant fuzzy information, and applied them to solve decision making problems. Xia et al. (2011) developed several series of aggregation operators for hesitant fuzzy information with the aid of quasi-arithmetic means, and gave a group decision making method under hesitant fuzzy environment based on the developed aggregation operators and the weight-determined technics. Rodriguez et al. (2012) introduced the concept of hesitant fuzzy linguistic term set (HFLTS) to increase the flexibility and richness of linguistic elicitation, and presented a multi-criteria linguistic decision-making model in which experts provide their assessments by using linguistic expressions based on comparative terms. Zhu et al. (2012a) introduced the dual hesitant fuzzy set (DHFS), and investigated some basic operations, properties and an extension principle for DHFSs. The results were illustrated by a practical example of group forecasting. Motivated by the ideal of prioritized aggregation operators (Yager 2008), Wei (2012) developed some prioritized aggregation operators for aggregating hesitant fuzzy information, and utilized these operators to develop some approaches to solve the hesitant fuzzy multiple attribute decision making problems in which the attributes are in different priority level. Zhu et al. (2012b) extended the geometric Bonferroni mean (GBM) to hesitant fuzzy environment, and defined a hesitant fuzzy geometric Bonferroni mean, and applied it to multi-criteria decision making. Yu et al. (2012) extended the generalized Bonferroni mean (GBM) to hesitant fuzzy environment and proposed the generalized hesitant fuzzy Bonferroni mean (GHFBM). Then they proposed an approach based on proposed operator for multiple criteria group decision making under hesitant fuzzy environment. 
The aforementioned hesitant aggregation operators (Xia, Xu 2011; Xu, Xia 2011b; Xia et al. 2011; Wei 2012; Zhu et al. 2012b; Yu et al. 2012) are mainly based on product triangular norm (t-norm) and its dual triangular conorm (t-conorm) (probabilistic sum), which are the most commonly used ones in decision making applications (Schweizer, Sklar 1983; Hájek 1998). The product and Einstein t-norms are two prototypical examples of the class of strict t-norms. For an intersection on FS, a good alternative to the product t-norm is the Einstein t-norm, which typically gives the same smooth approximations as the product t-norm. Equivalently, for an intersection on FS, a good alternative to probabilistic sum is the Einstein sum. Wang and Liu $(2011,2012)$ introduced Einstein operations on IFSs, and studied some intuitionistic fuzzy aggregation operators with the help of Einstein operations. However, it seems that in the literature there is little investigation on aggregation techniques using the Einstein operations for aggregating a collection of HFEs. In this paper, we shall develop some geometric aggregation operators based on Einstein t-norm and its dual t-conorm, and give an application of these operators to MAGDM. To do so, this paper is structured as follows. In Section 1, we briefly review some basic concepts related to HFSs and the existing geometric operators for aggregating HFEs. In Section 2, we introduce some Einstein operations on HFSs, and develop some novel geometric aggregation operators, such as the hesitant fuzzy Einstein weighted geometric $\left(\mathrm{HFWG}^{\varepsilon}\right)$ operator, hesitant fuzzy ordered Einstein weighted geometric $\left(\mathrm{HFOWG}^{\varepsilon}\right.$ ) operator, and hesitant fuzzy Einstein hybrid weighted geometric (HFHWG $\left.{ }^{\varepsilon}\right)$ operator, for aggregating a collection of HFEs. In addition, we make some comparisons between the proposed operator and ones proposed by Xia and Xu (2011). In Section 3, we apply the $\mathrm{HFWG}^{\varepsilon}$ operator to MAGDM with hesitant fuzzy information. In the last section, we have a conclusion.

\section{Preliminary}

The FS, an extension of the classical notion of set, was introduced by Zadeh (1965).

Definition 1. Let a set $X$ be fixed, a FS $F$ on $X$ is defined as:

$$
F=\left\{\left\langle x, \mu_{F}(x)\right\rangle \mid x \in X\right\},
$$

where $\mu_{F}$ is a mapping from $X$ to the closed interval $[0,1]$, and for each $x \in X, \mu_{F}(x)$ is called the degree of membership of $x$ in $X$.

The set theoretical operations have had an important role since in the beginning of FS theory. Starting from Zadeh's operations min and max many other operators were introduced in the fuzzy set literature (Zadeh 1965). All types of the particular operators were included in the general concepts of t-norms and t-conorms (Schweizer, Sklar 1983; Hájek 1998), which satisfy the requirements of the conjunction and disjunction operators, respectively. They are the most general families of binary functions that map the unit square into the unit interval, i.e. $T:[0,1]^{2} \rightarrow[0,1]$ and $S:[0,1]^{2} \rightarrow[0,1]$, and they are related by the De Morgan duality: the $\mathrm{t}$-conorm $S$ can be defined as $S(x, y)=1-T(1-x, 1-y)$, where $(x, y) \in[0,1]^{2}$. 
Here, we introduce some examples of the t-norms and t-conorms (Schweizer, Sklar 1983; Hájek 1998):

- Zadeh-intersection min is a t-norm, Zadeh-union max is a t-conorm;

- Algebraic product $\hat{x}$ is a t-norm and Algebraic sum $\hat{+}$ is a t-conorm, where:

$$
\hat{\imath}(x, y)=x y, \hat{+}(x, y)=x+y-x y ;
$$

- Einstein product $\dot{\varepsilon}$ is a t-norm and Einstein sum $\varepsilon$ is a t-conorm, where:

$$
\dot{\varepsilon}(x, y)=\frac{x y}{1+(1-x)(1-y)}, \quad \dot{\varepsilon}=\frac{x+y}{1+x y} .
$$

The most accepted one extension of the FS is the notion of IFS (Atanassov 1986), which is characterized by a membership function and a non-member function.

Definition 2. Let a set $X$ be fixed, an IFS $A$ in $X$ is defined as:

$$
A=\left\{\left\langle x, \mu_{A}(x), v_{A}(x)\right\rangle \mid x \in X\right\},
$$

where $\mu_{A}$ and $v_{A}$ are mappings from $X$ to the closed interval $[0,1]$ such that $0 \leq \mu_{A}(x) \leq 1$, $0 \leq v_{A}(x) \leq 1$ and $0 \leq \mu_{A}(x)+v_{A}(x) \leq 1$, for all $x \in X$, and they denote the degrees of membership and non-membership of element $x \in X$ to the set $A$, respectively. Let $\pi_{A}(x)=1-\mu_{A}(x)-v_{A}(x)$, then it is usually called the intuitionistic fuzzy index of element $x \in X$ to $A$, representing the degree of indeterminacy or hesitation of $x$ to $A$. It is obvious that $0 \leq \pi_{A}(x) \leq 1$ for $x \in X$.

However, when giving the membership degree of an element on FS, the difficulty of establishing the membership degree is not because we have a margin of error, or some possibility distribution on the possibility values, but because we have several possible values. For such cases, Torra and Narukawa (2009) and Torra (2010) proposed another generation of FS as follows.

Definition 3. Let $X$ be a reference set, then hesitant fuzzy set on $X$ is defined in terms of a function $h$ that when applied to $X$ returns a subset of $[0,1]$.

To be easily understood, Xia and Xu (2011) express the HFS as follows:

Definition 4. Let $X$ be a fixed set, a HFS $E$ on $X$ is defined as:

$$
E=\left\{\left\langle x, h_{E}(x)\right\rangle \mid x \in X\right\},
$$

where $h_{E}(x)$ is a set-valued function from $X$ to the power set of the unit interval (i.e. $2^{[0,1]}$ ) and denotes the possible membership degrees of the element $x \in X$ to the set $E$. For convenience, let $\Omega$ be the set of all HFSs on $X$.

Given $x \in X, h_{E}(x)$ is called as a hesitant fuzzy element (HFE) (Xia, Xu 2011), which simply denoted as $h=h(x)$. For convenience, let $H$ be the set of all HFEs on $X$.

For three HFEs $h, h_{1}, h_{2} \in H$, Torra (2010) and Xia and Xu (2011) defined some operations on them, which can be described as:
1) $h^{c}=\bigcup_{\gamma \in h}\{1-\gamma\}$;
2) $h_{1} \cup h_{2}=\bigcup_{\gamma_{1} \in h_{1}, \gamma_{2} \in h_{2}} \max \left\{\gamma_{1}, \gamma_{2}\right\}$;
3) $h_{1} \cap h_{2}=\bigcup_{\gamma_{1} \in h_{1}, \gamma_{2} \in h_{2}} \min \left\{\gamma_{1}, \gamma_{2}\right\}$; 
4) $h_{1} \oplus h_{2}=\bigcup_{\gamma_{1} \in h_{1}, \gamma_{2} \in h_{2}}\left\{\gamma_{1}+\gamma_{2}-\gamma_{1} \gamma_{2}\right\}$;

5) $h_{1} \otimes h_{2}=\bigcup_{\gamma_{1} \in h_{1}, \gamma_{2} \in h_{2}}\left\{\gamma_{1} \gamma_{2}\right\}$;

6) $\lambda h=\bigcup_{\gamma \in h}\left\{1-(1-\gamma)^{\lambda}\right\}$;

7) $h^{\lambda}=\bigcup_{\gamma \in h}\left\{\gamma^{\lambda}\right\}$.

To compare the HFEs, Xia and Xu (2011) defined the following comparison laws.

Definition 5. For a HFE $h, s(h)=\frac{1}{\# h} \sum_{\gamma \in h} \gamma$ is called the score function of $h$, where $\# h$ is the number of the elements in $h$. For two HFEs $h_{1}$ and $h_{2}$, if $s\left(h_{1}\right)>s\left(h_{2}\right)$, then $h_{1}>h_{2}$; if $s\left(h_{1}\right)=s\left(h_{2}\right)$, then $h_{1}=h_{2}$.

Based on the above algebraic operational laws of HFEs and Definition 5, Xia and Xu (2011) proposed some geometric aggregation operators for aggregating HFEs as listed below:

For a collection of $n$ HFEs $h_{j}(j=1,2, \cdots n)$.

The hesitant fuzzy weighted geometric (HFWG) operator:

$$
\begin{aligned}
& \operatorname{HFWG}_{\omega}\left(h_{1}, h_{2}, \cdots, h_{n}\right)=h_{1}^{\omega_{1}} \otimes h_{2}^{\omega_{2}} \otimes, \cdots, \otimes h_{n}^{\omega_{n}}= \\
& \bigcup_{\gamma_{1} \in h_{1}, \gamma_{2} \in h_{2}, \cdots, \gamma_{n} \in h_{n}}\left\{\prod_{j=1}^{n} \gamma_{j}^{\omega_{j}}\right\},
\end{aligned}
$$

where $\omega=\left(\omega_{1}, \omega_{2}, \cdots, \omega_{n}\right)^{T}$ is the weight vector of $h_{j}(j=1,2, \cdots n)$ with $\omega_{j} \in[0,1]$ and $\sum_{j=1}^{n} \omega_{j}=1$.

The hesitant fuzzy ordered weighted geometric (HFOWG) operator:

$$
\begin{aligned}
& \operatorname{HFOWG}_{w}\left(h_{1}, h_{2}, \cdots, h_{n}\right)=h_{\sigma(1)}^{\omega_{1}} \otimes h_{\sigma(2)}^{\omega_{2}} \otimes, \cdots, \otimes h_{\sigma(n)}^{\omega_{n}}= \\
& \bigcup_{\gamma_{\sigma(1)} \in h_{\sigma(1)}, \gamma_{\sigma(2)} \in h_{\sigma(2)}, \cdots, \gamma_{\sigma(n)} \in h_{\sigma(n)}}\left\{\prod_{j=1}^{n} \gamma_{\sigma(j)}^{\omega_{j}}\right\},
\end{aligned}
$$

where $h_{\sigma(j)}$ is the $j^{\text {th }}$ largest of $h_{k}(k=1,2, \cdots, n)$, and $w=\left(w_{1}, w_{2}, \cdots, w_{n}\right)^{T}$ is the aggregation-associated vector with $w_{j} \in[0,1]$ and $\sum_{j=1}^{n} w_{j}=1$.

The hesitant fuzzy hybrid weighted geometric $\left(\mathrm{HFHWG}^{\varepsilon}\right)$ operator:

$$
\begin{aligned}
& \operatorname{HFHWG}_{\omega, w}\left(h_{1}, h_{2}, \cdots, h_{n}\right)=\dot{h}_{\sigma(1)}^{\omega_{1}} \otimes \dot{h}_{\sigma(2)}^{\omega_{2}} \otimes, \cdots, \otimes \dot{h}_{\sigma(n)}^{\omega_{n}}= \\
& \bigcup_{\dot{\gamma}_{\sigma(1)} \in \dot{h}_{\sigma(1)} \dot{\gamma}_{\sigma(2)} \in \dot{h}_{\sigma(2)}, \cdots, \dot{\gamma}_{\sigma(n)} \in \dot{h}_{\sigma(n)}}\left\{\prod_{j=1}^{n} \dot{\gamma}_{\sigma(j)}^{\omega_{j}}\right\},
\end{aligned}
$$

where $\dot{h}_{\sigma(j)}$ is the $j^{\text {th }}$ largest of $\dot{h}_{k}\left(\dot{h}_{k}=h_{k}^{\left(n \omega_{k}\right)}, k=1,2, \cdots, n\right)$, and $\omega=\left(\omega_{1}, \omega_{2}, \cdots, \omega_{n}\right)^{T}$ is the weight vector of $h_{j}(j=1,2, \cdots n)$ with $\omega_{j} \in[0,1]$ and $\sum_{j=1}^{n} \omega_{j}=1 . w=\left(w_{1}, w_{2}, \cdots, w_{n}\right)^{T}$ is the aggregation-associated vector such that $w_{j} \in[0,1]$ and $\sum_{j=1}^{n} w_{j}=1$.

Note that the HFWG, HFOWG and HFHWG operators extend the WG, OWG, and HWG operators to aggregate HFEs, respectively. 


\section{Hesitant fuzzy Einstein geometric averaging aggregation operators}

In this section, we first introduce the extension principle for extending functions to HFEs proposed by Torra and Narukawa (2009).

Definition 6. Let $\Theta$ be a function $\Theta:[0,1]^{n} \rightarrow[0,1]$ and $\hbar=\left\{h_{1}, h_{2}, \cdots, h_{n}\right\}$ be a set of $n$ HFEs, then the extension of $\Theta$ on $\hbar$ is a function $\Theta_{\hbar}: H^{n} \rightarrow H$,

$$
\Theta_{\hbar}=\bigcup_{\gamma \in h_{1} \times h_{2} \times \cdots \times h_{n}}\{\Theta(\gamma)\},
$$

where $\Theta_{\hbar}$ the extension of an operator $\Theta$ on a set of HFEs $\hbar$, considers all the values in such sets and the application of $\Theta$ on them.

Naturally, this extension principle permits us to consider alternative operations for sum and product on HFEs, e.g. Einstein sum and Einstein product.

Let $\Theta$ be the Einstein operations on FSs, then the extensions of the Einstein sum and Einstein product on $h_{1}$ and $h_{2}$ (denoted by $h_{1} \oplus_{\varepsilon} h_{2}$ and $h_{1} \otimes_{\varepsilon} h_{2}$ respectively) are defined as follows respectively:

1) $\begin{aligned} & h_{1} \oplus_{\varepsilon} h_{2}=\bigcup_{\gamma_{1} \in h_{1}, \gamma_{2} \in h_{2}}\left\{\frac{\gamma_{1}+\gamma_{2}}{1+\gamma_{1} \gamma_{2}}\right\} \\ & \text { 2) } h_{1} \otimes_{\varepsilon} h_{2}=\bigcup_{\gamma_{1} \in h_{1}, \gamma_{2} \in h_{2}}\left\{\frac{\gamma_{1} \gamma_{2}}{1+\left(1-\gamma_{1}\right)\left(1-\gamma_{2}\right)}\right\} \text {. }\end{aligned}$

Theorem 1. If $n$ is any a positive integer and $h$ is a HFE of $H$, then the power multiplication operation $h^{n}{ }^{n}$ is a mapping from $Z^{+} \times H$ to $H$ :

$$
h_{\varepsilon}^{n}=\bigcup_{\gamma \in h}\left\{\frac{2 \gamma^{n}}{(2-\gamma)^{n}+\gamma^{n}}\right\},
$$

where: $h \cdot{ }_{\varepsilon}^{n}=\overbrace{h \otimes_{\varepsilon} h \otimes_{\varepsilon} \cdots \otimes_{\varepsilon} h}^{n}$.

Proof. Mathematical induction can be used to prove that Eq. (8) holds for all positive integers $n$. Eq. (8) is called $P(n)$.

Basis: Show that the statement $P(n)$ holds for $n=1$. The statement $P(n)$ amounts to the statement $P(1): h \varepsilon^{1}=\bigcup_{\gamma \in h}\left\{\frac{2 \gamma}{(2-\gamma)+\gamma}\right\}$.

In the left-hand side of the equation, $h \varepsilon^{1}=h=\bigcup_{\gamma \in h}\{\gamma\}$. In the right-hand side of the equation, $\bigcup_{\gamma \in h}\left\{\frac{2 \gamma}{(2-\gamma)+\gamma}\right\}=\bigcup_{\gamma \in h}\{\gamma\}$. The two sides are equal, so the statement $P(n)$ is true for $n=1$. Thus it has been shown the statement $P(1)$ holds.

Inductive step: Show that if $P(n)$ holds, then also $P(n+1)$ holds. Assume $P(n)$ holds (for some unspecified value of $n$ ). It must then be shown that $P(n+1)$ holds, that is: $h_{\varepsilon}{ }^{(n+1)}=\bigcup_{\gamma \in h}\left\{\frac{2 \gamma^{(n+1)}}{(2-\gamma)^{(n+1)}+\gamma^{(n+1)}}\right\}$. Using the induction hypothesis that $P(n)$ holds, the lefthand side can be rewritten to $h^{\cdot}{ }^{n} \otimes_{\varepsilon} h$ and based on the Einstein product operation of two HFEs, we have:

$$
h \cdot{ }^{n} \otimes_{\varepsilon} h=\bigcup_{\gamma \in h}\left\{\frac{\frac{2 \gamma^{n}}{(2-\gamma)^{n}+\gamma^{n}} \cdot \gamma}{1+\left(1-\frac{2 \gamma^{n}}{(2-\gamma)^{n}+\gamma^{n}}\right) \cdot(1-\gamma)}\right\}=\bigcup_{\gamma \in h}\left\{\frac{2 \gamma^{(n+1)}}{(2-\gamma)^{(n+1)}+\gamma^{(n+1)}}\right\} .
$$


Thereby showing that indeed $P(n+1)$ holds. Since both the basis and the inductive step have been proved, it has now been proved by mathematical induction that $P(n)$ holds for any positive integer $n$.

It can be easily verified that $0 \leq \frac{2 \gamma^{n}}{(2-\gamma)^{n}+\gamma^{n}} \leq 1$ even if $n$ is any positive real number. Thus, the operation $h^{n}{ }^{n}$ defined above is a HFS for any positive real number $n$.

Theorem 2. Let $h, h_{1}$ and $h_{2}$ be three HFEs and let $h_{3}=h_{1} \otimes_{\varepsilon} h_{2}$ and $h_{4}=h_{\varepsilon}{ }^{\lambda}, \lambda>0$, then both $h_{3}$ and $h_{4}$ are also HFEs.

In the following, let us look at $h_{\varepsilon} \lambda$ for some special cases of $\lambda$ and $h$.

Proposition 1. Let $h, h_{1}$ and $h_{2}$ be three HFEs, $\lambda, \lambda_{1}, \lambda_{2}>0$, then:

1) $h_{1} \otimes_{\varepsilon} h_{2}=h_{2} \otimes_{\varepsilon} h_{1}$;

2) $\left(h_{1} \otimes_{\varepsilon} h_{2}\right)^{\cdot \varepsilon^{\lambda}}=h_{1}^{\dot{\varepsilon}^{\lambda}} \otimes_{\varepsilon} h_{2}^{\dot{\varepsilon}^{\lambda}}$;

3) $h^{\cdot{ }^{\lambda_{1}}} \otimes_{\varepsilon} h^{\cdot \lambda_{2}}=h^{\cdot\left(\lambda_{1}+\lambda_{2}\right)}$;

4) $h^{\cdot{ }^{2} \lambda_{2} \lambda_{1}}=\left(h^{\cdot{ }^{2} \lambda_{2}}\right)^{\lambda_{1}}$.

\subsection{Hesitant fuzzy Einstein weighted geometric $\left(\mathrm{HFWG}^{\varepsilon}\right)$ operator}

Similar to the HFWG operator (i.e. (4) described in Section 1), the HFWG ${ }^{\varepsilon}$ operator is defined as follows:

Definition 7. Let $h_{j}(j=1,2, \cdots, n)$ be a collection of HFEs, then a hesitant fuzzy Einstein weighted geometric averaging $\left(\mathrm{HFWG}^{\varepsilon}\right)$ operator is a mapping $\mathrm{HFWG}^{\varepsilon}: H^{n} \rightarrow H$,

$$
\operatorname{HFWG}_{\omega}^{\varepsilon}\left(h_{1}, h_{2}, \ldots, h_{n}\right)=h_{1}^{\dot{\varepsilon}^{\omega}} \otimes_{\varepsilon} h_{2}^{\dot{\varepsilon}^{\omega}} \otimes_{\varepsilon}, \ldots, \otimes_{\varepsilon} h_{n} \omega_{n}^{\omega_{n}},
$$

where $\omega=\left(\omega_{1}, \omega_{2}, \ldots, \omega_{n}\right)^{T}$ is the weight vector of $h_{j}(j=1,2, \ldots, n)$ with $\omega_{j} \in[0,1]$ and $\sum_{j=1}^{n} \omega_{j}=1$.

Especially, if $\omega=\left(\frac{1}{n}, \frac{1}{n}, \ldots, \frac{1}{n}\right)^{T}$, then the $\mathrm{HFWG}^{\varepsilon}$ operator is reduced to a hesitant fuzzy Einstein geometric averaging $\mathrm{HFG}^{\varepsilon}$ operator of dimension $n$, which is defined as follows:

$$
\operatorname{HFG}_{\omega}^{\varepsilon}\left(h_{1}, h_{2}, \ldots, h_{n}\right)=\left(h_{1} \otimes_{\varepsilon} h_{2} \otimes_{\varepsilon}, \ldots, \otimes_{\varepsilon} h_{n}\right)^{\frac{1}{\varepsilon}} \text {. }
$$

With the Einstein operational laws of HFEs, the HFWG ${ }^{\varepsilon}$ operator, i.e. Eq. (9), can be transformed into the following form by induction on $n$.

Theorem 3. Let $h_{j}(j=1,2, \cdots, n)$ be a collection of HFEs, then their aggregated value by using the $\mathrm{HFWG}^{\varepsilon}$ operator is also a $\mathrm{HFE}$ and

$$
\operatorname{HFWG}_{\omega}^{\varepsilon}\left(h_{1}, h_{2}, \ldots, h_{n}\right)=\bigcup_{\gamma_{1} \in h_{1}, \gamma_{2} \in h_{2}, \cdots, \gamma_{n} \in h_{n}}\left\{\frac{2 \prod_{j=1}^{n} \gamma_{j}^{\omega_{j}}}{\prod_{j=1}^{n}\left(2-\gamma_{j}\right)^{\omega_{j}}+\prod_{j=1}^{n} \gamma_{j}^{\omega_{j}}}\right\},
$$

where $\omega=\left(\omega_{1}, \omega_{2}, \ldots, \omega_{n}\right)^{T}$ is the weight vector of $h_{j}(j=1,2, \ldots, n)$ such that $\omega_{j} \in[0,1]$, $j=1,2, \ldots, n$ and $\sum_{j=1}^{n} \omega_{j}=1$. 
Especially, if $\omega=\left(\frac{1}{n}, \frac{1}{n}, \ldots, \frac{1}{n}\right)^{T}$, then the $\mathrm{HFWG}^{\varepsilon}$ operator is reduced to the $\mathrm{HFG}^{\varepsilon}$ operator of dimension $n$, which is expressed as follows:

$$
\operatorname{HFG}_{\omega}^{\varepsilon}\left(h_{1}, h_{2}, \ldots, h_{n}\right)=\bigcup_{\gamma_{1} \in h_{1}, \gamma_{2} \in h_{2}, \cdots, \gamma_{n} \in h_{n}}\left\{\frac{2 \prod_{j=1}^{n} \gamma_{j}^{\frac{1}{n}}}{\prod_{j=1}^{n}\left(2-\gamma_{j}\right)^{\frac{1}{n}}+\prod_{j=1}^{n} \gamma_{j}^{\frac{1}{n}}}\right\} .
$$

Proof. The first result follows quickly from Theorem 2. Below we prove Eq. (11) by using mathematical induction on $n$. We first prove that Eq. (11) holds for $n=2$.

Since $h_{1}^{\dot{\varepsilon} \omega_{1}}=\bigcup_{\gamma_{1} \in h_{1}}\left\{\frac{2 \gamma_{1}^{\omega_{1}}}{\left(2-\gamma_{1}\right)^{\omega_{1}}+\gamma_{1}^{\omega_{1}}}\right\}$ and $h_{2}^{\varepsilon^{\omega}}=\bigcup_{\gamma_{2} \in h_{2}}\left\{\frac{2 \gamma_{2}^{\omega_{2}}}{\left(2-\gamma_{2}\right)^{\omega_{2}}+\gamma_{2}^{\omega_{2}}}\right\}$, and let $a_{1}=\gamma_{1}^{\omega_{1}}$, $b_{1}=\left(2-\gamma_{1}\right)^{\omega_{1}}, a_{2}=\gamma_{2}^{\omega_{2}}$ and $b_{2}=\left(2-\gamma_{2}\right)^{\omega_{2}}$, then:

$$
\begin{aligned}
& \operatorname{HFWG}_{\omega}^{\varepsilon}\left(h_{1}, h_{2}\right)=h_{1}^{\varepsilon^{\varepsilon} \omega_{1}} \otimes_{\varepsilon} h_{2}^{\varepsilon^{\omega} \omega_{2}}= \\
& \bigcup_{\gamma_{1} \in h_{1}}\left\{\frac{2 a_{1}}{b_{1}+a_{1}}\right\} \otimes_{\varepsilon} \cup_{\gamma_{2} \in h_{2}}\left\{\frac{2 a_{2}}{b_{2}+a_{2}}\right\}= \\
& \bigcup_{\gamma_{1} \in h_{1}, \gamma_{2} \in h_{2}}\left\{\frac{2 a_{1} a_{2}}{b_{1} b_{2}+a_{1} a_{2}}\right\}= \\
& \bigcup_{\gamma_{1} \in h_{1}, \gamma_{2} \in h_{2}}\left\{\frac{2 \gamma_{1}^{\omega_{1}} \gamma_{2}^{\omega_{2}}}{\left(2-\gamma_{1}\right)^{\omega_{1}}\left(2-\gamma_{2}\right)^{\omega_{2}}+\gamma_{1}^{\omega_{1}} \gamma_{2}^{\omega_{2}}}\right\},
\end{aligned}
$$

i.e. Eq. (11) holds for $n=2$.

If Eq. (11) holds for $n=k$, i.e.:

$$
\operatorname{HFWG}_{\omega}^{\varepsilon}\left(h_{1}, h_{2}, \ldots, h_{k}\right)=\bigcup_{\gamma_{1} \in h_{1}, \gamma_{2} \in h_{2}, \ldots, \gamma_{k} \in h_{k}}\left\{\frac{2 \prod_{j=1}^{k} \gamma_{j}^{\omega_{j}}}{\prod_{j=1}^{k}\left(2-\gamma_{j}\right)^{\omega_{j}}+\prod_{j=1}^{k} \gamma_{j}^{\omega_{j}}}\right\},
$$

then, when $n=k+1$, by the Einstein product, we have:

$$
\begin{aligned}
& \operatorname{HFWG}_{\omega}^{\varepsilon}\left(h_{1}, h_{2}, \ldots, h_{k+1}\right)= \\
& h_{1}^{\dot{\varepsilon} \omega_{1}} \otimes_{\varepsilon} h_{2}^{\dot{\varepsilon} \omega_{2}} \otimes_{\varepsilon}, \ldots, \otimes_{\varepsilon} h_{k}^{\dot{\varepsilon} \omega_{k}} \otimes_{\varepsilon} h_{k+1}^{\dot{\varepsilon} \omega_{k+1}}= \\
& \operatorname{HFWG}_{\omega}^{\varepsilon}\left(h_{1}, h_{2}, \ldots, h_{k}\right) \otimes_{\varepsilon} h_{k+1}^{\dot{\varepsilon} \omega_{k+1}}= \\
& \bigcup_{\gamma_{1} \in h_{1}, \gamma_{2} \in h_{2}, \ldots, \gamma_{k} \in h_{k}}\left\{\frac{2 \prod_{j=1}^{k} \gamma_{j}^{\omega_{j}}}{\prod_{j=1}^{k}\left(2-\gamma_{j}\right)^{\omega_{j}}+\prod_{j=1}^{k} \gamma_{j}^{\omega_{j}}}\right\} \otimes_{\varepsilon} U_{\gamma_{k+1} \in h_{k+1}}\left\{\frac{2 \gamma_{k+1}^{\omega_{k+1}}}{\left.\left(2-\gamma_{k+1}\right)^{\omega_{k+1}+\gamma_{k+1}^{\omega_{k+1}}}\right\},}\right.
\end{aligned}
$$

and let $a_{1}=\prod_{j=1}^{k} \gamma_{j}^{\omega_{j}}, b_{1}=\prod_{j=1}^{k}\left(2-\gamma_{j}\right)^{\omega_{j}}, a_{2}=\gamma_{k+1}^{\omega_{k+1}}$ and $b_{2}=\left(2-\gamma_{k+1}\right)^{\omega_{k+1}}$, then: 


$$
\begin{aligned}
& \operatorname{HFWG}_{\omega}^{\varepsilon}\left(h_{1}, h_{2}, \ldots, h_{k+1}\right)= \bigcup_{\gamma_{1} \in h_{1}, \gamma_{2} \in h_{2}, \ldots, \gamma_{k} \in h_{k}}\left\{\frac{2 a_{1}}{b_{1}+a_{1}}\right\} \otimes_{\varepsilon} \cup_{\gamma_{k+1} \in h_{k+1}}\left\{\frac{2 a_{2}}{b_{2}+a_{2}}\right\}= \\
& \bigcup_{\gamma_{1} \in h_{1}, \gamma_{2} \in h_{2}, \ldots, \gamma_{k} \in h_{k}, \gamma_{k+1} \in h_{k+1}}\left\{\frac{2 a_{1} a_{2}}{b_{1} b_{2}+a_{1} a_{2}}\right\}= \\
& \bigcup_{\gamma_{1} \in h_{1}, \gamma_{2} \in h_{2}, \ldots, \gamma_{k} \in h_{k}, \gamma_{k+1} \in h_{k+1}}\left\{\frac{2 \prod_{j=1}^{k+1} \gamma_{j}^{\omega_{j}}}{\prod_{j=1}^{k+1}\left(2-\gamma_{j}\right)^{\omega_{j}}+\prod_{j=1}^{k+1} \gamma_{j}^{\omega_{j}}}\right\},
\end{aligned}
$$

i.e. Eq. (11) holds for $n=k+1$.

Therefore, Eq. (11) holds for all $n$, which completes the proof of Theorem 3.

Lemma 4 (Torra, Narukawa 2007; Xu 2000). Let $a_{j}>0, \omega_{j}>0, j=1,2, \ldots, n$ and $\sum_{j=1}^{n} \omega_{j}=1$, then:

$$
\prod_{j=1}^{n} a_{j}^{\omega_{j}} \leq \sum_{j=1}^{n} \omega_{j} a_{j}
$$

with equality if and only if $a_{1}=a_{2}=\cdots=a_{n}$.

Corollary 1. The HfWG operator and the $\mathrm{HFWG}^{\varepsilon}$ operator have the following relation:

$$
\operatorname{HFWG}_{\omega}^{\varepsilon}\left(h_{1}, h_{2}, \ldots, h_{n}\right) \geq \operatorname{HFWG}_{\omega}\left(h_{1}, h_{2}, \ldots, h_{n}\right) \text {, }
$$

where $h_{j}(j=1,2, \ldots, n)$ be a collection of HFEs and $\omega=\left(\omega_{1}, \omega_{2}, \ldots, \omega_{n}\right)^{T}$ is the weight vector of $h_{j}(j=1,2, \ldots, n)$, with $\omega_{j} \in[0,1], j=1,2, \ldots, n$ and $\sum_{j=1}^{n} \omega_{j}=1$.

Proof. For $\gamma_{1} \in h_{1}, \gamma_{2} \in h_{2}, \ldots, \gamma_{n} \in h_{n}$, based on Lemma 4, we have:

$$
\prod_{j=1}^{n}\left(2-\gamma_{j}\right)^{\omega_{j}}+\sum_{j=1}^{n} \gamma_{j}^{\omega_{j}} \leq \sum_{j=1}^{n} \omega_{j}\left(2-\gamma_{j}\right)+\sum_{j=1}^{n} \omega_{j} \gamma_{j}=2 \text {, }
$$

then:

$$
\frac{2 \prod_{j=1}^{n} \gamma_{j}^{\omega_{j}}}{\prod_{j=1}^{n}\left(2-\gamma_{j}\right)^{\omega_{j}}+\prod_{j=1}^{n} \gamma_{j}^{\omega_{j}}} \geq \prod_{j=1}^{n} \gamma_{j}^{\omega_{j}},
$$

where that equality holds if and only if $\gamma_{1}=\gamma_{2}=\cdots=\gamma_{n}$.

Corollary 1 shows that the values obtained by the $\mathrm{HFWG}^{\varepsilon}$ operator are bigger than the ones obtained by the HFWG operator.

Example 1. Let $h_{1}=\{0.2,0.3,0.5\}, h_{2}=\{0.4,0.6\}$ be two HFEs, and $\omega=(0.7,0.3)^{T}$ be the weight vector of $h_{j}(j=1,2)$, then by Theorem 3 , we have:

$$
\begin{aligned}
& \operatorname{HFWG}_{\omega}^{\varepsilon}\left(h_{1}, h_{2}\right)=\bigcup_{\gamma_{1} \in h_{1}, \gamma_{2} \in h_{2}}\left\{\frac{2 \gamma_{1}^{\omega_{1}} \gamma_{2}^{\omega_{2}}}{\left(2-\gamma_{1}\right)^{\omega_{1}}\left(2-\gamma_{2}\right)^{\omega_{2}}+\gamma_{1}^{\omega_{1}} \gamma_{2}^{\omega_{2}}}\right\}= \\
& \{0.2482,0,2856,0.3276,0.3744,0.4683,0.5288\} .
\end{aligned}
$$


If we use the HFWG operator developed by Xia and Xu (2011) (i.e. (4) described in Section 1), to aggregate the HFEs $h_{j}(j=1,2)$, then we have:

$$
\begin{aligned}
& \operatorname{HFWG}_{\omega}\left(h_{1}, h_{2}\right)=\bigcup_{\gamma_{1} \in h_{1}, \gamma_{2} \in h_{2}}\left\{\gamma_{1}^{\omega_{1}} \gamma_{2}^{\omega_{2}}\right\}= \\
& \{0.2462,0,2781,0.3270,0.3693,0.4676,0.5281\} .
\end{aligned}
$$

\subsection{Hesitant fuzzy Einstein ordered weighted geometric $\left(\mathrm{HFOWG}^{\varepsilon}\right)$ operator}

Similar to the definition of the HFOWG operator (i.e. Eq. (5) described in Section 1), in what follows, we develop a hesitant fuzzy Einstein ordered weighted geometric (HFOWG $)$ operator.

Definition 8. Let $h_{j}(j=1,2, \ldots, n)$ be a collection of HFEs, a HFOWG ${ }^{\varepsilon}$ operator of dimension $n$ is a mapping HFOWG $: H^{n} \rightarrow H$ that has an associated vector $w=\left(w_{1}, w_{2}, \ldots, w_{n}\right)^{T}$ such that $w_{j} \in[0,1], j=1,2, \ldots, n$ and $\sum_{j=1}^{n} w_{j}=1$, and

$$
\operatorname{HFOWG}_{w}^{\varepsilon}\left(h_{1}, h_{2}, \ldots, h_{n}\right)=h_{\sigma(1)}^{\dot{w_{1}}} \otimes_{\varepsilon} h_{\sigma(2)}^{\dot{w_{2}}} \otimes_{\varepsilon}, \ldots, \otimes_{\varepsilon} h_{\sigma(n)}^{\dot{\varepsilon} w_{n}},
$$

where $(\sigma(1), \sigma(2), \ldots, \sigma(n))$ is a permutation of $(1,2, \ldots, n)$ such that $h_{\sigma(j-1)} \geq h_{\sigma(j)}$ for all $j=2,3, \ldots, n$. Especially, if $w=\left(\frac{1}{n}, \frac{1}{n}, \ldots, \frac{1}{n}\right)^{T}$, then the $\mathrm{HFOWG}^{\varepsilon}$ operator is reduced to the $\mathrm{HFG}^{\varepsilon}$ operator of dimension $n$.

Based on Definition 5 and the Einstein operational laws of HFEs, the HFOWG ${ }^{\varepsilon}$ operator (15) can be transformed into the following form.

Theorem 5. Let $h_{j}(j=1,2, \ldots, n)$ be a collection of HFEs, then their aggregated value by using the $\mathrm{HFOWG}^{\varepsilon}$ operator is also an $\mathrm{HFE}$, and

$$
\begin{aligned}
& \operatorname{HFOWG}_{w}^{\varepsilon}\left(h_{1}, h_{2}, \ldots, h_{n}\right)= \\
& \bigcup_{\gamma_{\sigma(1)} \in h_{\sigma(1)}, \gamma_{\sigma(2)} \in h_{\sigma(2)}, \cdots, \gamma_{\sigma(n)} \in h_{\sigma(n)}}\left\{\frac{2 \prod_{j=1}^{n} \gamma_{\sigma(j)}^{w_{j}}}{\prod_{j=1}^{n}\left(2-\gamma_{\sigma(j)}\right)^{w_{j}}+\prod_{j=1}^{n} \gamma_{\sigma(j)}^{w_{j}}}\right\},
\end{aligned}
$$

where $(\sigma(1), \sigma(2), \ldots, \sigma(n))$ is a permutation of $(1,2, \ldots, n)$ such that $h_{\sigma(j-1)} \geq h_{\sigma(j)}$ for all $j=2,3, \ldots, n . w=\left(w_{1}, w_{2}, \ldots, w_{n}\right)^{T}$ is the weighting vector of the HFOWG ${ }^{\varepsilon}$ operator such that $w_{j} \in[0,1], j=1,2, \ldots, n$ and $\sum_{j=1}^{n} w_{j}=1$.

Note that the HFOWG and $\mathrm{HFOWG}^{\varepsilon}$ operators are developed based on the idea of the OWA operator (Yager 1988). The main characterization of the OWA operator is its reordering step. Several methods have been developed to obtain the OWA weights. Yager (1988) used linguistic quantifiers to compute the OWA weights. O'Hagan (1988) generated the OWA weights with a predefined degree of orness by maximizing the entropy of the OWA weights. Filev and Yager (1998) obtained the OWA weights based on the exponential smoothing. Yager and Filev (1999) got the OWA weights from a collection of samples with the relevant aggregated data. Xu and $\mathrm{Da}$ (2002) obtained the OWA weights under partial weight information by establishing a linear objective-programming model. Especially, based on the normal distribution (Gaussian distribution), $\mathrm{Xu}$ (2005) developed a method to obtain the 
OWA weights, whose prominent characteristic is that it can relieve the influence of unfair arguments on the decision result by assigning low weights to those "false" or "biased" ones.

Corollary 2. The HFOWG and $\mathrm{HFOWG}^{\varepsilon}$ operators have the following relation:

$$
\operatorname{HFOWG}_{w}^{\varepsilon}\left(h_{1}, h_{2}, \ldots, h_{n}\right) \geq \operatorname{HFOWG}_{w}\left(h_{1}, h_{2}, \ldots, h_{n}\right),
$$

where $h_{j}(j=1,2, \ldots, n)$ are a collection of HFEs and $w=\left(w_{1}, w_{2}, \ldots, w_{n}\right)^{T}$ is the weight vector of $h_{j}(j=1,2, \ldots, n)$ with $w_{j} \in[0,1], j=1,2, \ldots, n$ and $\sum_{j=1}^{n} w_{j}=1$.

Proof. Similar to Corollary 1.

Example 2. Let $h_{1}=\{0.1,0.4\}, h_{2}=\{0.3,0.5\}$ and $h_{3}=\{0.2,0.5,0.8\}$ be three HFEs. And the $\mathrm{HFOWG}^{\varepsilon}$ operator has an associated vector $w=(0.25,0.4,0.35)^{T}$.

By Definition 5, we calculate the scores $s\left(h_{j}\right)$ of $h_{j}(j=1,2,3)$ :

$$
s\left(h_{1}\right)=0.25, s\left(h_{2}\right)=0.4, s\left(h_{3}\right)=0.5 \text {. }
$$

Since $s\left(h_{3}\right)>s\left(h_{2}\right)>s\left(h_{1}\right)$, then:

$$
h_{\sigma(1)}=h_{3}, h_{\sigma(2)}=h_{2}, h_{\sigma(3)}=h_{1} .
$$

Thus by Eq. (16), it follows that:

$\operatorname{HFOWG}_{w}^{\varepsilon}\left(h_{1}, h_{2}, h_{3}\right)=$

$\bigcup_{\gamma_{\sigma(1)} \in h_{\sigma(1)}, \gamma_{\sigma(2)} \in h_{\sigma(2)}, \gamma_{\sigma(3)} \in h_{\sigma(3)}}\left\{\frac{2 \prod_{j=1}^{3} \gamma_{\sigma(j)}^{w_{j}}}{\prod_{j=1}^{3}\left(2-\gamma_{\sigma(j)}\right)^{w_{j}}+\prod_{j=1}^{n} \gamma_{\sigma(j)}^{w_{j}}}\right\}=$

$\{0.1866,0.2344,0.2386,0.2775,0.2974,0.3016,0.3440,0.3727,0.3789,0.4350,0.4632,0.5277\}$.

If we use the HFOWG operator, developed by Xia and Xu (2011) (i.e. (5) described in Section 1), to aggregate the HFEs $h_{j}(j=1,2,3)$, then we have:

$\operatorname{HFOWG}_{w}\left(h_{1}, h_{2}, h_{3}\right)=$

$\bigcup_{\gamma_{\sigma(1)} \in h_{\sigma(1)}, \gamma_{\sigma(2)} \in h_{\sigma(2)}, \gamma_{\sigma(3)} \in h_{\sigma(3)}}\left\{\prod_{j=1}^{3} \gamma_{\sigma(j)}^{w_{j}}\right\}=$

$\{0.1845,0.2264,0.2321,0.2610,0.2847,0.2998,0.3202,0.3678,0.3770,0.4240,0.4624,0.5201\}$.

\subsection{Hesitant fuzzy Einstein hybrid weighted geometric $\left(\mathrm{HFHWG}^{\varepsilon}\right)$ operator}

The HFWG ${ }^{\varepsilon}$ operator weights the hesitant fuzzy argument itself. The HFOWG ${ }^{\varepsilon}$ operator weights the values instead of weighting the arguments. This is so because each $\omega_{j}$ is attached to the $j^{\text {th }}$ value in decreasing order without considering from which argument the value comes. Therefore, weights represent different aspects in both aggregation operators. However, although both points of view are meaningful in a single problem, both aggregation operators present the drawback of considering only one of them. To solve this drawback, it 
is necessary to introduce a hybrid aggregation operator for hesitant fuzzy arguments, which considers the importance of the arguments and the importance of the values of the arguments.

Definition 9. Let $h_{j}(j=1,2, \ldots, n)$ be a collection of HFEs, the HFHWG ${ }^{\varepsilon}$ operator of dimension $n$ is a mapping HFHWG $H^{\varepsilon} \rightarrow H$ that has an associated vector $w=\left(w_{1}, w_{2}, \ldots, w_{n}\right)^{T}$ such that $w_{j} \in[0,1], j=1,2, \ldots, n$ and $\sum_{j=1}^{n} w_{j}=1$, then:

$$
\operatorname{HFHWG}_{\omega, w}^{\varepsilon}\left(h_{1}, h_{2}, \ldots, h_{n}\right)=\dot{h}_{\sigma(1)}^{\varepsilon} \otimes_{\varepsilon} \otimes_{\varepsilon} \dot{h_{\sigma}^{\varepsilon} w_{2}} \otimes_{\varepsilon}, \ldots, \otimes_{\varepsilon} \dot{h}_{\sigma(n)}^{\varepsilon} w_{n},
$$

where: $\dot{h}_{\sigma(j)}$ is the $j^{\text {th }}$ largest of the weighted HFEs $\dot{h}_{i}\left(\dot{h}_{i}=h_{i}^{\dot{\varepsilon}}\left(n \omega_{i}\right), i=1,2, \ldots, n\right)$; $\omega=\left(\omega_{1}, \omega_{2}, \ldots, \omega_{n}\right)^{T}$ is the weight vector of $h_{j}(j=1,2, \ldots, n)$ with $\omega_{j} \in[0,1], j=1,2, \ldots, n$ and $\sum_{j=1}^{n} \omega_{j}=1$, and $n$ is the balancing coefficient.

Similar to Theorem 5, the $\mathrm{HFHWG}^{\varepsilon}$ operator (18) can be transformed into the following form.

Theorem 6. Let $h_{j}(j=1,2, \ldots, n)$ be a collection of HFEs. A hesitant fuzzy Einstein hybrid averaging $\mathrm{HFHWG}^{\varepsilon}$ operator of dimension $n$ is a mapping $\mathrm{HFHWG}^{\varepsilon}: H^{n} \rightarrow H$ that has an associated vector $w=\left(w_{1}, w_{2}, \ldots, w_{n}\right)^{T}$ such that $w_{j} \in[0,1], j=1,2, \ldots, n$ and $\sum_{j=1}^{n} w_{j}=1$, then:

$$
\begin{aligned}
& \operatorname{HFHWG}_{\omega, w}^{\varepsilon}\left(h_{1}, h_{2}, \ldots, h_{n}\right)= \\
& \bigcup_{\dot{\gamma}_{\sigma(1)} \in \dot{h}_{\sigma(1)}, \dot{\gamma}_{\sigma(2)} \in \dot{h}_{\sigma(2)}, \cdots, \dot{\gamma}_{\sigma(n)} \in \dot{h}_{\sigma(n)}}\left\{\frac{2 \prod_{j=1}^{n} \dot{\gamma}_{\sigma(j)}^{w_{j}}}{\prod_{j=1}^{n}\left(2-\dot{\gamma}_{\sigma(j)}\right)^{w_{j}}+\prod_{j=1}^{n} \dot{\gamma}_{\sigma(j)}^{w_{j}}}\right\},
\end{aligned}
$$

where: $\dot{h}_{\sigma(j)}$ is the $j^{\text {th }}$ largest of the weighted HFEs $\dot{h}_{i}\left(\dot{h}_{i}=h_{i}^{\left.\varepsilon^{\left(n \omega_{i}\right.}\right)}, i=1,2, \ldots, n\right)$; $\omega=\left(\omega_{1}, \omega_{2}, \ldots, \omega_{n}\right)^{T}$ is the weight vector of $h_{j}(j=1,2, \ldots, n)$ with $\omega_{j} \in[0,1], j=1,2, \ldots, n$ and $\sum_{j=1}^{n} \omega_{j}=1$; and $n$ is the balancing coefficient.

Corollary 3. The $\mathrm{HFWG}^{\varepsilon}$ operator is a special case of the $\mathrm{HFHWG}^{\varepsilon}$ operator. Proof. If $w=\left(\frac{1}{n}, \frac{1}{n}, \ldots, \frac{1}{n}\right)^{T}$, then:

$$
\begin{aligned}
& \operatorname{HFHWG}_{\omega, w}^{\varepsilon}\left(h_{1}, h_{2}, \ldots, h_{n}\right)=\dot{h}_{\sigma(1)}^{w_{1}} \otimes_{\varepsilon} \dot{\dot{h}_{\sigma(2)}^{\varepsilon} w_{2}} \otimes_{\varepsilon}, \ldots, \otimes_{\varepsilon} \dot{h}_{\sigma(n)}^{\varepsilon w_{n}}= \\
& \ddot{h}_{\sigma(1)}^{\varepsilon^{\frac{1}{n}}} \otimes_{\varepsilon} \ddot{h}_{\sigma(2)}^{\dot{\varepsilon}^{\frac{1}{n}}} \otimes_{\varepsilon}, \ldots, \otimes_{\varepsilon} \ddot{h}_{\sigma(n)}^{\dot{\varepsilon}_{n}^{\frac{1}{n}}}=h_{\sigma(1)}^{\dot{\omega_{1}}} \otimes_{\varepsilon} h_{\sigma(2)}^{\dot{\varepsilon} \omega_{2}} \otimes_{\varepsilon}, \ldots, \otimes_{\varepsilon} h_{\sigma(n)}^{\dot{\omega}^{\omega_{n}}}= \\
& \operatorname{HFWG}_{\omega}^{\varepsilon}\left(h_{1}, h_{2}, \ldots, h_{n}\right) \text {, }
\end{aligned}
$$

which completes the proof of Corollary 3.

Corollary 4. The HFOWG ${ }^{\varepsilon}$ operator is a special case of the $\mathrm{HFHWG}^{\varepsilon}$ operator. Proof. If $\omega=\left(\frac{1}{n}, \frac{1}{n}, \ldots, \frac{1}{n}\right)^{T}$, then:

$$
\begin{aligned}
& \operatorname{HFHWG}_{\omega, w}^{\varepsilon}\left(h_{1}, h_{2}, \ldots, h_{n}\right)=\dot{h_{\sigma(1)}^{\varepsilon} w_{1}} \otimes_{\varepsilon} \dot{h_{\sigma(2)}^{\varepsilon} w_{2}} \otimes_{\varepsilon}, \ldots, \otimes_{\varepsilon} \dot{h_{\sigma(n)}^{\varepsilon}}= \\
& h_{\sigma(1)}^{w^{w_{1}}} \otimes_{\varepsilon} \dot{h_{\sigma(2)}^{w_{2}}} \otimes_{\varepsilon}, \ldots, \otimes_{\varepsilon} \dot{h}_{\sigma(n)}^{\dot{w_{n}}}= \\
& \operatorname{HFOWG}_{w}^{\varepsilon}\left(h_{1}, h_{2}, \ldots, h_{n}\right),
\end{aligned}
$$

which completes the proof of Corollary 4 . 
Example 3. Let $h_{1}=\{0.2,0.4,0.5\}, h_{2}=\{0.2,0.6\}$ and $h_{3}=\{0.1,0.3,0.4\}$ are three HFEs, and $\omega=(0.15,0.30,0.55)^{T}$ be the weight vector of $h_{j}(j=1,2,3)$, the HFHWG ${ }^{\varepsilon}$ operator has an associated vector $w=(0.3,0.4,0.3)^{T}$.

We first get the weighted hesitant fuzzy values $\dot{h}_{j}=h_{j}^{\cdot\left(3 \omega_{j}\right)}(j=1,2,3)$ :

$$
\begin{aligned}
& \dot{h}_{1}={\dot{h_{1}^{\varepsilon}}}^{\left(3 \omega_{1}\right)}=\{0.5423,0.6978,0.7577\}, \\
& \dot{h_{2}}={\dot{h_{2}^{\varepsilon}}}^{\dot{\varepsilon}^{\left(3 \omega_{2}\right)}}=\{0.2432,0.6362\}, \\
& \dot{h}_{3}=\dot{h}_{3}^{\varepsilon\left(3 \omega_{3}\right)}=\{0.0154,0.1081,0.1843\},
\end{aligned}
$$

and the scores $s\left(\dot{h}_{j}\right)$ of $\dot{h}_{j}(j=1,2,3)$ :

$$
s\left(\dot{h}_{1}\right)=0.6659, s\left(\dot{h}_{2}\right)=0.4397, s\left(\dot{h}_{3}\right)=0.1026 .
$$

Since $s\left(\dot{h}_{1}\right)>s\left(\dot{h}_{2}\right)>s\left(\dot{h}_{3}\right)$, then:

$$
\dot{h}_{\sigma(1)}=\dot{h}_{1}, \dot{h}_{\sigma(2)}=\dot{h}_{2}, \dot{h}_{\sigma(3)}=\dot{h}_{3} .
$$

By Eq. (19), it follows that:

$\operatorname{HFHWG}_{\omega, w}^{\varepsilon}\left(h_{1}, h_{2}, h_{3}\right)=\bigcup_{\dot{\gamma}_{\sigma(1)} \in \dot{h}_{\sigma(1)}, \dot{\gamma}_{\sigma(2)} \in \dot{h}_{\sigma(2)}, \dot{\gamma}_{\sigma(3)} \in \dot{h}_{\sigma(3)}}\left\{\frac{2 \prod_{j=1}^{3} \dot{\gamma}_{\sigma(j)}^{w_{j}}}{\prod_{j=1}^{3}\left(2-\dot{\gamma}_{\sigma(j)}\right)^{w_{j}}+\prod_{j=1}^{3} \dot{\gamma}_{\sigma(j)}^{w_{j}}}\right\}=$

$\{0.1455,0.1610,0.1668,0.2262,0.2491,0.2499,0.2577,0.2749,0.2842$,

$0.2901,0.3183,0.3289,0.3768,0.4115,0.4243,0.4324,0.4707,0.4848\}$.

\section{Decision making based on hesitant fuzzy information}

The MAGDM problems are widespread in real life decision situations. In some practical problems, such as presidential election or the blind peer review of thesis, the experts propose the preferences or opinions for the alternatives with anonymous in order to protect their privacy or avoid influencing each other. In such situations, HFE permits us to represent the rating of the alternative on the attribute given by several experts, so we use a hesitant fuzzy decision matrix to describe the group decision making problems. Suppose there are $n$ alternatives $X=\left\{x_{1}, x_{2}, \ldots, x_{n}\right\}$ and $n$ attributes $G=\left\{g_{1}, g_{2}, \ldots, g_{m}\right\}$ with the attribute weight vector $\omega=\left(\omega_{1}, \omega_{2}, \ldots, \omega_{m}\right)^{T}$ such that $\omega_{j} \in[0,1], j=1,2, \ldots, m$ and $\sum_{j=1}^{m} \omega_{j}=1$. The ratings of alternatives $x_{i}(i=1,2, \ldots, n)$ on attributes $g_{j}(j=1,2, \ldots, m)$ given by the experts are HFEs $h_{i j}(i=1,2, \ldots, n ; j=1,2, \ldots, m)$, where $h_{i j}$ indicates the set of the degrees that the alternative $x_{i}$ satisfies the attribute $g_{j}$ given by the experts. In the case where more than one experts provide the same value, then the value emerges only once in $h_{i j}$. Hence, a fuzzy MAGDM problem can be concisely expressed in matrix format: $D=\left(h_{i j}\right)_{n \times m}$ (Table 1$)$. 
Table 1 . The hesitant fuzzy decision matrix $D$

\begin{tabular}{cccccc}
\hline & $g_{1}$ & $g_{2}$ & $\cdots$ & $g_{m}$ \\
\hline$x_{1}$ & $h_{11}$ & $h_{12}$ & $\cdots$ & $h_{1 m}$ \\
\hline$x_{2}$ & $h_{21}$ & $h_{22}$ & $\cdots$ & $h_{2 m}$ \\
\hline$\vdots$ & $\vdots$ & $\vdots$ & $\ddots$ & $\vdots$ \\
\hline$x_{n}$ & $h_{n 1}$ & $h_{n 2}$ & $\cdots$ & $h_{n m}$ \\
\hline
\end{tabular}

In what follows, we apply the $\mathrm{HFWG}^{\varepsilon}$ and HFWG operators proposed by Xia and Xu (2011) to hesitant fuzzy MAGDM, which involves the following steps.

Step 1. Obtain the normalized hesitant fuzzy decision matrix. In general, attributes can be classified into two types: benefit attributes and cost attributes. In other words, the attribute set $G$ can be divided into two subsets: $G_{1}$ and $G_{2}$, which are the subset of benefit attributes and cost attributes, respectively. Furthermore, $G_{1} \cup G_{2}=G$ and $G_{1} \cap G_{2}=\Phi$, where $\Phi$ is empty set. The decision matrix $D$ needs to be normalized besides all the attributes $g_{j}(j=1,2, \ldots, m)$ are of the same type. In this paper we choose the following normalization formula to update the hesitant fuzzy decision matrix $D$ :

$$
h_{i j}=\left\{\begin{array}{ll}
h_{i j} & j \in G_{1} \\
h_{i j}^{c} & j \in G_{2}
\end{array},\right.
$$

where $h_{i j}^{c}$ is the complement of $h_{i j}$. Hence, we obtain the normalized hesitant fuzzy decision matrix $D=\left(h_{i j}\right)_{n \times m}$.

Step 2. Compute the overall ratings of alternatives. Utilize the $\mathrm{HFWG}^{\varepsilon}$ operator (or the HFWG operator) to aggregate all the rating values $h_{i j}(j=1,2, \ldots, m)$ of the $i^{\text {th }}$ line and get the overall rating value $h_{i}$ corresponding to the alternative $x_{i}(i=1,2, \ldots, n)$, i.e.:

$$
h_{i}=\operatorname{HFWG}_{w}^{\varepsilon}\left(h_{i 1}, h_{i 2}, \ldots, h_{i m}\right), i=1,2, \ldots, n \text {. }
$$

Step 3. Rank the order of all alternatives. Utilize the method in Definition 5 to compute the scores of the overall rating values $h_{i}(i=1,2, \ldots, n)$, and rank all the alternatives $x_{i}(i=1,2, \ldots, n)$ in accordance with $h_{i}(i=1,2, \ldots, n)$ in descending order, finally select the most desirable alternative(s) with the largest overall rating value.

We consider a MAGDM problem involves the prioritization of a set of information technology improvement projects (adapted from (Ngwenyama, Bryson 1999)) is used to illustrate the developed $\mathrm{HFWG}^{\varepsilon}$ operator.

Example 4. The information management steering committee of Midwest American Manufacturing Company must prioritize for development and implementation a set of four information technology improvement projects $X=\left\{x_{1}, x_{2}, x_{3}, x_{4}\right\}: x_{1}$ - Quality Management Information, $x_{2}$ - Customer Order Tracking, $x_{3}$ - Inventory Control and $x_{4}$ - Budget Analysis. 
The committee is concerned that the projects are prioritized from highest to lowest potential contribution to the firm's strategic goal of gaining competitive advantage in the industry. In assessing the potential contribution of each project, three factors are considered as follows (it should be noted that three factors are benefit attributes):

- Productivity $\left(g_{1}\right)$ : The productivity factor assesses the potential of a proposed project to increase effectiveness and efficiency of the firm's manufacturing and service operations;

- Differentiation $\left(g_{2}\right)$ : The differentiation factor assesses the potential of a proposed project to fundamentally differentiate the firm's products and services from its competitors, and to make them more desirable to its customers;

- Management $\left(g_{3}\right)$ : The management factor assesses the potential of a proposed project to assist management in improving their planning, controlling and decision-making activities.

Additionally suppose that the weight vector of the attributes is:

$\omega=\left(\omega_{1}, \omega_{2}, \omega_{3}\right)^{T}=(0.45,0.25,0.3)^{T}$.

In order to avoid influencing each other, the members of the committee are required to provide their preferences in anonymity, so the committee, which includes five members, represents the characteristics of the projects $x_{i}(i=1,2,3,4)$ by the HFEs $h_{i j}(i=1,2,3,4 ; j=1,2,3)$ with respect to the factors $g_{j}(j=1,2,3)$, listed in Table 2 .

Table 2. The hesitant fuzzy decision matrix $D$

\begin{tabular}{cccc}
\hline & $g_{1}$ & $g_{2}$ & $g_{3}$ \\
\hline$x_{1}$ & $\{0.2,0.4,0.7\}$ & $\{0.2,0.6,0.8\}$ & $\{0.2,0.3,0.6,0.7\}$ \\
\hline$x_{2}$ & $\{0.2,0.4,0.7,0.9\}$ & $\{0.1,0.2,0.4,0.5\}$ & $\{0.3,0.4,0.6,0.9\}$ \\
\hline$x_{3}$ & $\{0.3,0.5,0.6,0.7\}$ & $\{0.2,0.4,0.5,0.6\}$ & $\{0.3,0.5,0.7,0.8\}$ \\
\hline$x_{4}$ & $\{0.3,0.5,0.6\}$ & $\{0.2,0.4\}$ & $\{0.5,0.6,0.7\}$ \\
\hline
\end{tabular}

In the following, we use the developed $\mathrm{HFWG}^{\varepsilon}$ operator to get the optimal project.

Step 1. Because all the factors are benefit attributes, the hesitant fuzzy decision matrix $D$ need not be updated.

Step 2. Utilize the $\mathrm{HFWG}^{\varepsilon}$ operator to aggregate all the rating values $h_{i j}(j=1,2,3)$ of the $i^{\text {th }}$ line and get the overall rating value $h_{i}$ corresponding to the alternative $x_{i}(i=1,2,3,4)$. Due to vast amounts of data, we only give $h_{4}$ as an instance, the results of $h_{1}, h_{2}$ and $h_{3}$ are not listed.

$$
\begin{aligned}
& h_{4}=\operatorname{HFWG}_{\omega}^{\varepsilon}\left(h_{41}, h_{42}, h_{43}\right)=\bigcup_{\gamma_{41} \in h_{41}, \gamma_{42} \in h_{42}, \gamma_{43} \in h_{43}}\left\{\frac{2 \prod_{j=1}^{3} \gamma_{4 j}^{\omega_{j}}}{\prod_{j=1}^{3}\left(2-\gamma_{4 j}\right)^{\omega_{j}}+\prod_{j=1}^{3} \gamma_{4 j}^{\omega_{j}}}\right\}= \\
& \{0.3197,0.3404,0.3602,0.3779,0.4016,0.4042,0.4240,0.4290,0.4419, \\
& 0.4526,0.4684,0.4735,0.4934,0.5013,0.5156,0.5275,0.5450,0.5725\} .
\end{aligned}
$$


Step 3. Compute the scores of the overall rating values $h_{i}(i=1,2,3,4)$ by Definition 5 , and rank all the alternatives $x_{i}(i=1,2,3,4)$ in accordance with $h_{i}(i=1,2,3,4)$ in descending order. The scores of the overall rating values and the rankings of alternatives are listed in Table 3. Finally select the most desirable alternative $x_{2}$ with the largest overall rating value.

Table 3. The overall scores and rankings of alternatives

\begin{tabular}{cccccc}
\hline & $x_{1}$ & $x_{2}$ & $x_{3}$ & $x_{4}$ & Ranking \\
\hline HFWG $^{\varepsilon}$ & 0.4354 & 0.4503 & 0.4995 & 0.4472 & $x_{3} \succ x_{2} \succ x_{4} \succ x_{1}$ \\
\hline HFWG & 0.4252 & 0.4343 & 0.4924 & 0.4405 & $x_{3} \succ x_{4} \succ x_{2} \succ x_{1}$ \\
\hline
\end{tabular}

In Step 2, if we use the HFWG operator proposed by Xia and Xu (2011) (i.e. (4) described in Section 1) to aggregation the values of the alternatives, then the scores of the overall rating values $h_{i}(i=1,2,3,4)$ and the rankings of the alternatives $x_{i}(i=1,2,3,4)$ are also listed in Table 3.

From Table 3, we can see that the values obtained by the $\mathrm{HFWG}^{\varepsilon}$ operator are always greater than the ones obtained by the HFWG operator for the same aggregation rating values corresponding to the alternatives, but the rankings of alternatives by both different aggregation operators are slightly different, and the best projects are the same, i.e. the project $x_{3}$.

Remark 1. Using the HFWG ${ }^{\varepsilon}$ operator rather than the HFWG operator, the decision-maker has more optimistic attitude in aggregation process. Using different techniques reflects the decision maker's optimistic (or pessimistic) attitude in aggregation process. Therefore, in general, the different aggregation operators do not always return the same ranking orders and the same alternatives.

\section{Conclusion}

Motivated by the extension principle of HFSs, we have extended the Einstein operations on FSs to HFSs, and have developed some new hesitant fuzzy aggregation operators, including the $\mathrm{HFWG}^{\varepsilon}$ operator, $\mathrm{HFOWG}^{\varepsilon}$ operator, and $\mathrm{HFHWG}^{\varepsilon}$ operator. Then, we have applied the $\mathrm{HFWG}^{\varepsilon}$ to the DMGDM problem with anonymity. It is worth point out that these aggregation operators are the same effective tools as the aggregation operators proposed by Xia and $\mathrm{Xu}$ (2011), for aggregating hesitant fuzzy information. It is worth pointing that the proposed aggregation operators not only have the same good nature as those proposed by Xia and $\mathrm{Xu}$ (2011), but also can reflect the decision-makers more optimistic attitude in aggregation process.

\section{Acknowledgment}

This work was supported by the National Natural Science Foundation of China (NSFC) (71171048, 71371049), the PhD Program Foundation of Chinese Ministry of Education (20120092110038), 
the Scientific Research and Innovation Project for College Graduates of Jiangsu Province (CXZZ11_0185), the Scientific Research Foundation of Graduate School of Southeast University (YBJJ1135) and the State Key Laboratory of Rail Traffic Control and Safety (RCS2011K002) of Beijing Jiaotong University.

\section{References}

Aczl, J.; Saaty, T. L. 1983. Procedures for synthesizing ratio judgments, Journal of Mathematical Psychology 27(1): 93-102. http://dx.doi.org/10.1016/0022-2496(83)90028-7

Atanassov, K.; Gargov, G. 1989. Interval valued intuitionistic fuzzy sets, Fuzzy Sets and Systems 31(3): 343-349. http://dx.doi.org/10.1016/0165-0114(89)90205-4

Atanassov, K. T. 1986. Intuitionistic fuzzy sets, Fuzzy Sets and Systems 20(1): 87-96. http://dx.doi.org/10.1016/S0165-0114(86)80034-3

Beliakov, G.; Pradera, A.; Calvo, T. 2007. Aggregation functions: a guide for practitioners. Heidelberg, Berlin, New York: Springer. $361 \mathrm{p}$.

Benjamin, C. O.; Ehie, I. C.; Omurtag, Y. 1992. Planning facilities at the university of Missouri-rolla, Interfaces 22(4): 95-105. http://dx.doi.org/10.1287/inte.22.4.95

Calvo, T.; Mayor, G.; Mesiar, R. 2002. Aggregation operators: new trends and applications. Heidelberg, Germany: Physica-Verlag. 352 p. http://dx.doi.org/10.1007/978-3-7908-1787-4

Chakrabarty, K.; Despi, I. 2007. $n^{k}$-bags, International Journal of Intelligent Systems 22(2): 223-236. http://dx.doi.org/10.1002/int.20195

Chiclana, F.; Herrera, F.; Herrera-Viedma, E. 2001. Integrating multiplicative preference relations in a multipurpose decision-making model based on fuzzy preference relations, Fuzzy Sets and Systems 122(2): 277-291. http://dx.doi.org/10.1016/S0165-0114(00)00004-X

Cornelis, C.; Deschrijver, G.; Kerre, E. E. 2004. Implication in intuitionistic fuzzy and interval-valued fuzzy set theory: construction, classification, application, International Journal of Approximate Reasoning 35(1): 55-95. http://dx.doi.org/10.1016/S0888-613X(03)00072-0

Dubois, D.; Gottwald, S.; Hájek, P.; Kacprzyk, J.; Prade, H. 2005. Terminological difficulties in fuzzy set theory - the case of "intuitionistic fuzzy sets", Fuzzy Sets and Systems 156(3): 485-491. http://dx.doi.org/10.1016/j.fss.2005.06.001

Dubois, D.; Prade, H. M. 1980. Fuzzy sets and systems: theory and applications. Academic Press. 393 p.

Filev, D.; Yager, R. R. 1998. On the issue of obtaining OWA operator weights, Fuzzy Sets and Systems 94(2): 157-169. http://dx.doi.org/10.1016/S0165-0114(96)00254-0

Grabisch, M.; Marichal, J.-L.; Mesiar, R.; Pap, E. 2009. Aggregation functions. Cambridge: Cambridge University Press. 460 p. http://dx.doi.org/10.1017/CBO9781139644150

Hájek, P. 1998. Metamathematics of fuzzy logic. The Netherlands, Dordrecht: Kluwer Academic Publishers. 299 p. http://dx.doi.org/10.1007/978-94-011-5300-3

Herrera, F.; Herrera-Viedma, E.; Chiclana, F. 2001. Multiperson decision-making based on multiplicative preference relations, European Journal of Operational Research 129(2): 372-385. http://dx.doi.org/10.1016/S0377-2217(99)00197-6

Mizumoto, M.; Tanaka, K. 1976. Some properties of fuzzy sets of type 2, Information and Control 31(4): 312-340. http://dx.doi.org/10.1016/S0019-9958(76)80011-3

Ngwenyama, O. K.; Bryson, N. 1999. Eliciting and mapping qualitative preferences to numeric rankings in group decision making. European Journal of Operational Research 116(3): 487-497.

http://dx.doi.org/10.1016/S0377-2217(98)00081-2 
O'Hagan, M. 1988. Aggregating template or rule antecedents in real-time expert systems with fuzzy set logic, in Proc. of the Twenty-Second Asilomar Conference on Signals, Systems and Computers, 31 October - 2 November, 1988, Pacific Grove, California, 2: 681-689. http://dx.doi.org/10.1109/ACSSC.1988.754637

Rodriguez, R. M.; Martinez, L.; Herrera, F. 2012. Hesitant fuzzy linguistic term sets for decision making, IEEE Transactions on Fuzzy Systems 20(1): 109-119. http://dx.doi.org/10.1109/TFUZZ.2011.2170076

Saaty, T. L. 1980. The analytic hierarchy process. New York: McGraw-Hill. 287 p.

Schweizer, B.; Sklar, A. 1983. Probabilistic metric spaces. New York: North Holland. 275 p.

Torra, V. 2010. Hesitant fuzzy sets, International Journal of Intelligent Systems 25(6): 529-539.

Torra, V.; Narukawa, Y. 2007. Modeling decisions: information fusion and aggregation operators. Germany, Berlin: Springer-Verlag. 298 p.

Torra, V.; Narukawa, Y. 2009. On hesitant fuzzy sets and decision, in Proc. of 2009 IEEE International Conference on Fuzzy Systems (FUZZ-IEEE 2009), 20-24 August, 2009, Jeju Island, Korea, 1378-1382. http://dx.doi.org/10.1109/FUZZY.2009.5276884

Wang, W.; Liu, X. 2011. Intuitionistic fuzzy geometric aggregation operators based on Einstein operations, International Journal of Intelligent Systems 26(11): 1049-1075. http://dx.doi.org/10.1002/int.20498

Wang, W.; Liu, X. 2012. Intuitionistic fuzzy information aggregation using Einstein operations, IEEE Transactions on Fuzzy Systems 20(5): 923-938. http://dx.doi.org/10.1109/TFUZZ.2012.2189405

Wei, G. 2012. Hesitant fuzzy prioritized operators and their application to multiple attribute decision making, Knowledge-Based Systems 31: 176-182. http://dx.doi.org/10.1016/j.knosys.2012.03.011

Willett, K.; Sharda, R. 1991. Using the analytic hierarchy process in water resources planning: selection of flood control projects, Socio-Economic Planning Sciences 25(2): 103-112. http://dx.doi.org/10.1016/0038-0121(91)90008-F

Xia, M.; Xu, Z. S. 2011. Hesitant fuzzy information aggregation in decision making, International Journal of Approximate Reasoning 52(3): 395-407. http://dx.doi.org/10.1016/j.ijar.2010.09.002

Xia, M.; Xu, Z. S.; Chen, N. 2011. Some hesitant fuzzy aggregation operators with their application in group decision making, Group Decision and Negotiation, 1-21.

$\mathrm{Xu}, \mathrm{Z}$. S. 2000. On consistency of the weighted geometric mean complex judgement matrix in AHP, European Journal of Operational Research 126(3): 683-687. http://dx.doi.org/10.1016/S0377-2217(99)00082-X

$\mathrm{Xu}, \mathrm{Z}$. S. 2005. An overview of methods for determining OWA weights, International Journal of Intelligent Systems 20(8): 843-865. http://dx.doi.org/10.1002/int.20097

$\mathrm{Xu}, \mathrm{Z}$. S.; Da, Q. L. 2002. The uncertain OWA operator, International Journal of Intelligent Systems 17(6): 569-575. http://dx.doi.org/10.1002/int.10038

$\mathrm{Xu}$, Z. S.; Da, Q. L. 2003. An overview of operators for aggregating information, International Journal of Intelligent Systems 18(9): 953-969. http://dx.doi.org/10.1002/int.10127

Xu, Z. S.; Xia, M. 2011a. Distance and similarity measures for hesitant fuzzy sets, Information Sciences 181(11): 2128-2138. http://dx.doi.org/10.1016/j.ins.2011.01.028

Xu, Z. S.; Xia, M. 2011b. On distance and correlation measures of hesitant fuzzy information, International Journal of Intelligent Systems 26(5): 410-425. http://dx.doi.org/10.1002/int.20474

Yager, R. R. 1986. On the theory of bags, International Journal of General Systems 13(1): 23-37. http://dx.doi.org/10.1080/03081078608934952

Yager, R. R. 1988. On ordered weighted averaging aggregation operators in multicriteria decision-making, IEEE Transactions on Systems Man and Cybernetics 18(1): 183-190. http://dx.doi.org/10.1109/21.87068

Yager, R. R. 2008. Prioritized aggregation operators, International Journal of Approximate Reasoning 48(1): 263-274. http://dx.doi.org/10.1016/j.ijar.2007.08.009 
Yager, R. R.; Filev, D. P. 1999. Induced ordered weighted averaging operators, IEEE Transactions on Systems, Man, and Cybernetics, Part B: Cybernetics 29(2): 141-150. http://dx.doi.org/10.1109/3477.752789

Yager, R. R.; Kacprzyk, J. 1997. The ordered weighted averaging operator: theory and applications. MA, Boston: Kluwer. 343 p. http://dx.doi.org/10.1007/978-1-4615-6123-1

Yu, D.; Wu, Y.; Zhou, W. 2012. Generalized hesitant fuzzy bonferroni mean and its application in multi-criteria group decision making, Journal of Information and Computational Science 9(2): 267-274.

Zadeh, L. A. 1965. Fuzzy sets, Information and Control 8(3): 338-353. http://dx.doi.org/10.1016/S0019-9958(65)90241-X

Zadeh, L. A. 1973. Outline of a new approach to analysis of complex systems and decision processes interval-valued fuzzy sets, IEEE Transactions on Systems Man and Cybernetics SMC3(1): 28-44. http://dx.doi.org/10.1109/TSMC.1973.5408575

Zhu, B.; Xu, Z. S.; Xia, M. 2012a. Dual hesitant fuzzy sets, Journal of Applied Mathematics 2012: 1-13. http://dx.doi.org/10.1155/2012/879629

Zhu, B.; Xu, Z. S.; Xia, M. 2012b. Hesitant fuzzy geometric bonferroni means, Information Sciences 205: 72-85. http://dx.doi.org/10.1016/j.ins.2012.01.048

Weize WANG received the BS degree in Computing Mathematics from Sichuan University, Chengdu, China, in 2001, the MS degree in System Engineering from Xiamen University, Xiamen, China, in 2009 and the PhD degree in Management Science and Engineering from Southeast University, Nanjing, China, in 2013. He is currently a Lecturer with the School of Economics and Management, Guangxi Normal University, Guilin, China. His research interests include fuzzy information fusion and aggregation operators and their application to multiple criteria decision making.

Xinwang LIU received the BS degree in Mathematics from Henan Normal University, Xinxiang, China, in 1991 and the MS and PhD degrees in Systems Engineering from Southeast University, Nanjing, China, in 1996 and 1999, respectively, where he is currently a Professor with the Department of Management Science and Engineering, School of Economics and Management. He is the author or coauthor of more than 40 publications. His research interests include type-2 fuzzy logic, aggregation operators, fuzzy decision making, and their applications. 